\title{
Propostas para revisão dos critérios clínicos de morte encefálica
}

\author{
Clinical features of brain death - review \\ and purposals
}

\begin{abstract}
Almir Ferreira de Andrade', Robson Luis Oliveira de Amorim², Wellingson Silva Paiva ${ }^{3}$, Eberval Gadelha Figueiredo ${ }^{4}$, Leonardo Borges de Barros e Silva ${ }^{5}$, Manoel Jacobsen Teixeira ${ }^{6}$
\end{abstract}

\begin{abstract}
Andrade AF de, Amorim RLO de, Paiva WS, Figueiredo EG, Barros e Silva LB de, Teixeira MJ. Propostas para revisão dos critérios clínicos de morte encefálica. Rev Med (São Paulo). 2007 jul.-set.;86(3):132-7.

RESUMO: Atualmente, quase 70 mil pessoas aguardam doação de órgãos no Brasil. O Hospital das Clínicas da Universidade de São Paulo - HCFMUSP é referência em transplantes de órgãos e tecidos e, ao mesmo tempo que se realizam cirurgias de alta complexidade, capacita os profissionais de saúde, sendo um facilitador para a integração dos mesmos. A morte encefálica hoje deve ser considerada uma emergência médica, pois pode salvar várias vidas. O seu diagnóstico é realizado de acordo com a Resolução do Conselho Federal de Medicina $\mathrm{n}^{\circ}$ 1.480/97 e, baseado nessa resolução e em nossa experiência com todo o processo de captação até o transplante, apresentamos neste artigo propostas para evitar a perda de potenciais doadores.
\end{abstract}

DESCRITORES: Morte encefálica/diagnóstico. Transplante de órgãos/legislação \& jurisprudência. Transplante de órgãos/normas.

\footnotetext{
1. Prof. Livre docente e Coordenador da Equipe de Neurocirurgia de Emergência, Hospital das Clínicas da Faculdade de Medicina da Universidade de São Paulo.

2. Divisão de Neurocirurgia, Hospital das Clínicas da Faculdade de Medicina da Universidade de São Paulo.

3. Divisão de Neurocirurgia, Hospital das Clínicas da Faculdade de Medicina da Universidade de São Paulo.

4. Divisão de Neurocirurgia, Hospital das Clínicas da Faculdade de Medicina da Universidade de São Paulo.

5. Coordenador da Organização de Procura de Órgãos, Hospital das Clínicas da Faculdade de Medicina da Universidade de São Paulo.

6. Professor Titular da Disciplina de Neurocirurgia, Hospital das Clínicas da Faculdade de Medicina da Universidade de São Paulo.

Endereço para correspondência: Wellingson Silva Paiva. Rua Dr. Ovídio Pires de Campos, 171. Ap. 511. CEP: 05403010. São Paulo, SP, Brasil. e-mail: wellingsonpaiva@yahoo.com.br
} 


\section{INTRODUÇÃO}

O Hospital das Clínicas da Faculdade de Medicina da Universidade de São Paulo - HCFMUSP tem uma longa tradição em transplantes de órgãos. Este hospital realizou o primeiro transplante cardíaco na América Latina, apenas três dias após o primeiro transplante cardíaco no mundo na Cidade do Cabo - África do Sul. Também escreveu história ao realizar o primeiro transplante de intestino delgado do mundo. Junto com o progresso da cirurgia de transplante, foi natural que se desenvolvesse o que se tornaria hoje uma ciência: o diagnóstico de morte encefálica. A hipótese diagnóstica de morte cerebral pode ser feita na sala de emergência, na sala de admissão de pronto-socorro e unidades de terapia intensiva com a finalidade de após confirmado este diagnóstico, segundo o protocolo do Conselho Federal de Medicina, orientar a conduta médica e oferecer informações aos familiares.

\section{Considerações gerais}

Diversos autores observaram hipóxia sistêmica, súbita diminuição da pressão arterial sistêmica e arritmia cardíaca letal em $26 \%$ e hipotensão arterial sistêmica em $39 \%$ dos pacientes submetidos ao teste de apnéia, reportando ainda a necessidade de critérios para a sua contra-indicação $0^{1,2}$.

Os pacientes em morte encefálica estão todos sob assistência ventilatória e freqüentemente apresentam complicações pulmonares, tais como aumento da produção de secreção, shunts pulmonares ou distúrbios de membrana alveolar ${ }^{3}$.

Estes aspectos podem causar problemas quando da realização do teste de apnéia. Não há dúvidas que novas rotinas podem ser incorporadas ao teste.

O exame neurológico com teste de apnéia e os exames complementares para determinação de morte encefálica podem ser feitos somente após condições clínicas favoráveis dos pacientes potenciais doadores de órgãos.

Considerando que o diagnóstico de morte encefálica para a doação de órgãos possui implicações filosóficas, religiosas e sociais especiais, temos preocupação em achar um consenso e oficializar questões referentes ao protocolo do teste de apnéia na morte encefálica, avaliação dos distúrbios metabólicos e a relevância do exame complementar na confirmação do diagnóstico de morte encefálica. Portanto, fundamentado no estudo realizado no centro de proteção sistêmica e de diagnóstico de morte encefálica, pro- pomos modificações nos parâmetros utilizados para definição de casos de morte encefálica no Brasil.

\section{DIAGNÓSTICO DE MORTE ENCEFÁLICA: IMPOR- TÂNCIA DE UMA UTI ESPECIALIZADA}

Desde 1998 que a enfermagem da OPO-HC tem se especializado na assistência a estes pacientes, com as orientações dos médicos intensivistas da UTI da Nefrologia ( 1 leito), $6^{\circ}$ andar Instituto Central do Hospital das Clínicas (ICHC). O número de paradas cardíacas entre os potenciais doadores durante 0 teste de apnéia (8 a 10\% em 1998 e 1999, nenhuma após 2000) e após as provas de morte encefálica (6 a 25\% entre 2000 e 2002, 1\% em 2003) reduziu sensivelmente.

Em fevereiro de 2000, foi implantada a Unidade de Proteção Sistêmica e Diagnóstico de Morte Encefálica, no $\mathrm{ICHC}, 5^{\circ}$ andar, sala 5103 (2 leitos), anexa à UTI neurológica sob cuidados de enfermagem da OPO e prescrição dos médicos dessa UTI.

A partir de 28 de agosto de 2003 , os dois leitos foram incluídos na Unidade de Terapia Intensiva da Cirurgia do Trauma, a unidade 4138 específica para proteção sistêmica e diagnóstico de morte encefálica, sob cuidados da mesma equipe da OPO, enfermagem da UTI do trauma, proteção sistêmica pela equipe de médicos da mesma UTI e diagnóstico neurológico de morte encefálica pela equipe médica neurocirúrgica de emergência. Essa idéia teve como base os novos artigos que mostram um aumento na sobrevida e mesmo melhoria do ponto de vista cientifico, ético e humanístico com a utilização de unidades especializadas em Terapia Intensiva Neurológica.

É necessário ainda modificar o ponto de vista de alguns profissionais. É difícil estabelecer que os pacientes com morte encefálica, e especialmente aqueles declarados doadores devem receber 0 máximo de atenção e cuidados. Alguns médicos e outros profissionais de saúde podem pensar que eles devem cuidar "dos vivos", apenas. Assim atuando, prejudicam muitos outros pacientes, ainda na espera para um transplante.

Os pacientes são admitidos em nosso hospital como potenciais doadores, após autorização da família para a doação de órgãos. Estes pacientes são admitidos em unidades de preservação dos parâmetros fisiológicos que muitas vezes podem carecer de equipamentos ou mesmo de equipe médica. Entretanto o diagnóstico de morte encefálica ainda não foi completado. Mas, uma vez que a morte encefálica não é confirmada, todos os esforços médicos devem ser realizados para a manutenção da vida (Figura 1). 
No figura 1 foram tomadas medidas clínicas e cirúrgicas para a manutenção da vida em uma Unidade de Terapia Intensiva, foi realizado o primeiro exame neurológico que foi compatível com morte encefálica. Antes da realização do segundo exame e da prova de apnéia, foi feito um SPECT que foi incompatível com morte encefálica. Assim, não foi feito o teste de apnéia e como o paciente já estava em UTI, foram mantidas as medidas para manutenção da vida. Além disso, o ambiente de UTI é mais propício ao tratamento e à manutenção destes pacientes. Devem ser desencorajados termos como "leitos de doação" ou "leitos de Morte Encefálica", que podem carecer de cuidados adequados em caso de não confirmação de Morte Encefálica e sim, leitos em UTI especializada.

\section{Propostas}

1. Pacientes potenciais doadores, já autorizados pela família para a doação de órgãos, internados na admissão do Pronto-Socorro que já tiverem realizado o primeiro exame neurológico devem ser transferidos para a Unidade de Proteção Sistêmica e Diagnóstico de Morte Encefálica a fim de que seja estabelecido o diagnóstico definitivo de Morte Encefálica, mantendo-se adequadamente seus parâmetros vitais.

2. Se a morte encefálica não se confirmar, obviamente permanecem todos os esforços médicos para a manutenção da vida, caso contrário seguese o fluxograma para se concretizar a doação dos órgãos ${ }^{4}$.

\section{A PROVA DE APNÉIA NA MORTE ENCEFÁLICA}

Hayashi e Takasu ${ }^{5}$ em 1998 estabeleceram que a prova de apnéia é um fator capaz de determinar distúrbios cardiovasculares, incluindo hipóxia sistêmica, hipotensão arterial sistêmica e arritmias letais, sendo observada em até $26 \%$ durante o teste de apnéia.

Compreendemos que o diagnóstico de morte encefálica só esteja completo até que as provas clínicas e exames complementares estejam realizados ${ }^{6}$. Portanto em teoria, a realização da prova de apnéia é um fator de aumento da morbi-mortalidade em um paciente gravemente doente ainda sem o diagnóstico de morte encefálica.

A Lei que sanciona o diagnóstico de morte encefálica não determina em que momento desta avaliação seja realizado o exame confirmatório ${ }^{7}$. $\mathrm{Na}$ Revista da Sociedade Brasileira de Neurocirurgia, o Dr. Luís Renato Mello em seu artigo intitulado "Ética na morte cerebral: pontos obscuros"8, discorre que
"Resta finalmente a pergunta: tomados todos os cuidados de pré-oxigenação, será que um teste de apnéia, realizado sem prévio teste confirmatório, não acaba de matar?."

A Equipe Médica de Emergência do PSNC do HC-FMUSP, apresentou o trabalho "Angiografia isotópica encefálica com Tc ${ }^{99 m}$ ECD e SPECT levantando que este exame poderia preceder o teste de apnéia nos pacientes com diagnóstico clínico de morte encefálica"9 no $12^{\circ}$ Congresso Mundial de Neurocirurgia, realizado em Sydney, Austrália, em 2001, no qual foi discutido o uso do SPECT ou outro exame confirmatório antes da realização do teste de apnéia em pacientes que apresentavam movimentos lazaróides e dúvidas quanto ao coma aperceptivo e arreativo ${ }^{10}$. Duas situações possíveis foram identificadas: ausência total de perfusão vascular e celular encefálica (Figura 2), compatível com morte encefálica; ou mostrar presença de perfusão celular encefálica (Figura 1), situação que contra-indica o teste de apnéia.

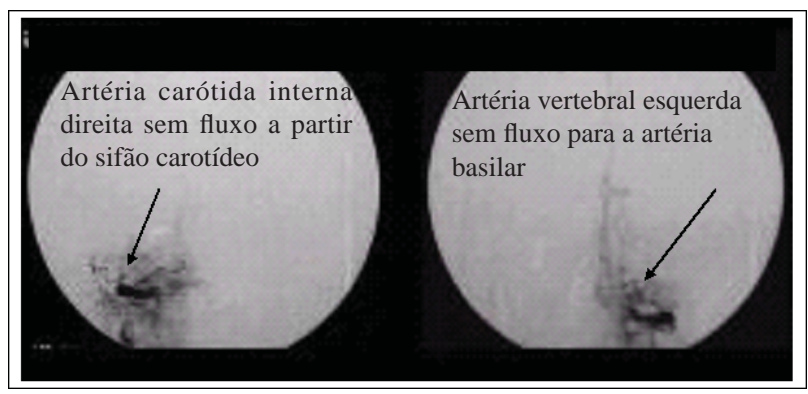

Figura 1. Angiografia digital dos territórios carotídeo e vértebro-basilar usada como teste confirmatório do diagnóstico de morte encefálica, 12 horas após o SPECT do exemplo 1
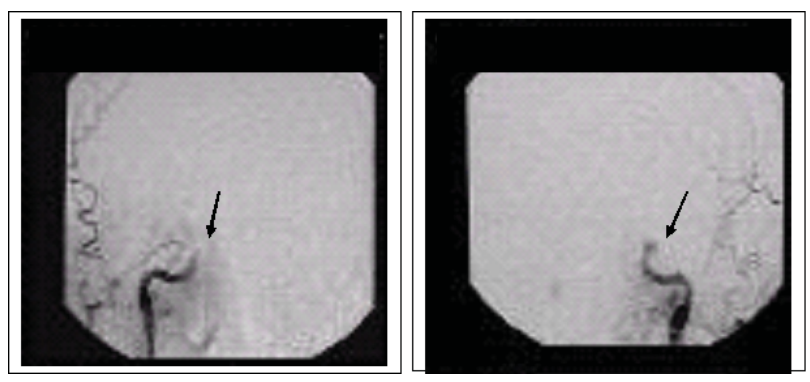

Figura 2. Paciente de 40 anos, masculino, ECGla $=3$ pontos, reflexo fotomotor ausente bilateralmente, movimentos inespecíficos no membro superior esquerdo. Teste confirmatório precedendo o teste de apnéia foi realizado (diagnóstico diferencial do movimento lazaróide). Tanto a angiografia cerebral isotópica quanto o SPECT demonstraram perfusão encefálica 
O critério japonês para diagnóstico da morte encefálica também recomenda a obtenção de um EEG isoelétrico antes da realização da prova de apnéia, fato refutado por Wijdicks ${ }^{1,10}$ que acredita que a função bulbar só pode ser detectada através do teste de apnéia, e não através da atividade elétrica detectada pelo EEG. Entretanto, Schwarz ${ }^{11}$ descreve situação de lesão primária do tronco cerebral em que a atividade elétrica cortical estava presente e o teste de apnéia poderia determinar hipertensão intracraniana e herniação. Em nosso estudo ${ }^{9}$, demonstramos, no entanto, que o exame do SPECT pode preencher esse argumento, já que em 14 pacientes com exame neurológico de coma aperceptivo e arreativo não houve demonstração de perfusão do tronco cerebral, confirmada por teste subseqüente de apnéia. Dessa forma, indicamos a realização do SPECT antes do teste de apnéia em caso de dúvida no exame neurológico (movimentos lazaróides ou inespecíficos) ${ }^{12}$, exame protocolar incompleto (pacientes externos ad- mitidos apenas com um ou dois exames neurológicos sem o exame complementar), dúvida no resultado de exame complementar operador-dependente ou lesão primária demonstrável em tronco cerebral (com permanência de atividade cortical) ${ }^{11}$.

\section{Propostas}

1. Realizar exame subsidiário inócuo - SPECT cerebral, Doppler transcraniano (Figura 1) ou potencial evocado de tronco cerebral (Figura 2) - antes da realização do teste de apnéia em casos de dúvida no exame neurológico (considerar movimentos involuntários referidos ou diagnosticados como de origem não medular para que eticamente possa se realizar exames confirmatórios antes do teste) $)^{9,12}$, protocolo considerado incompleto, dúvida em exame complementar operador-dependente ou presença de lesão primária do tronco cerebral.

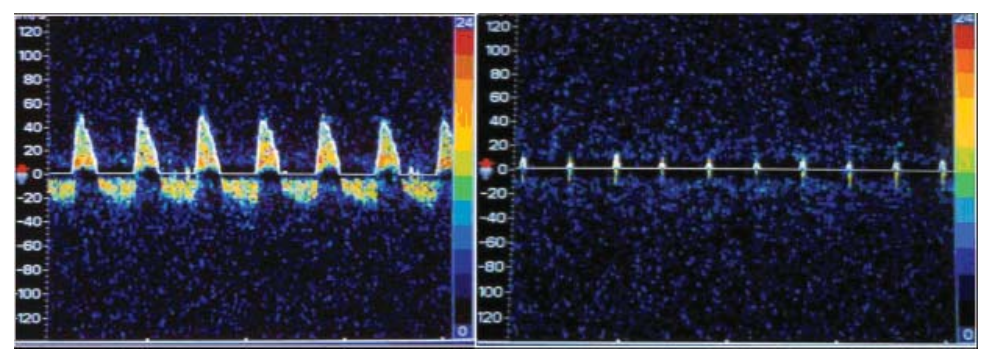

Figura 3. Doppler transcraniano evidenciando colapso circulatório encefálico total, à dir: FSC com presença de reversão diastólica; à esq: Espículas sistólicas.

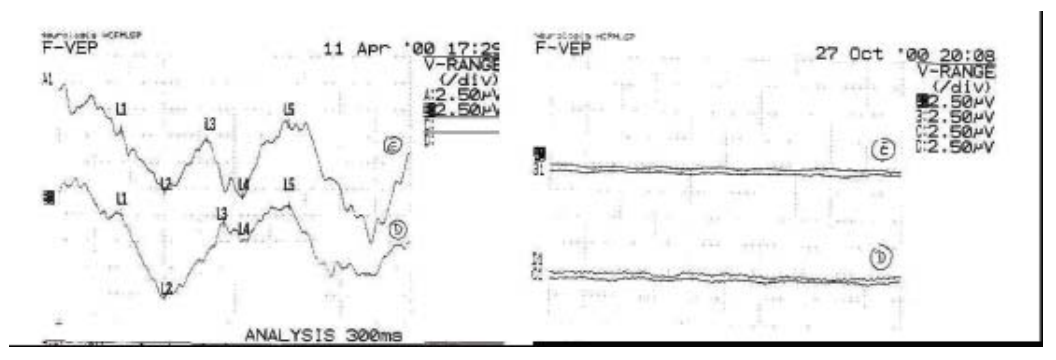

Figura 2. Avaliação por Potencial Evocado Visual (PEV) para complementação diagnóstica de morte encefálica. À dir. PEV em morte encefálica; à esq. PEV normal.

2. Iniciar o teste de apnéia com valores imediatos de gasometria arterial, com $\mathrm{PaCO}_{2}$ entre $35 \mathrm{e}$ $45 \mathrm{mmHg}$, reduzindo o tempo de observação para 6 minutos (considerados suficientes para atingir o ponto apnéico de $55 \mathrm{mmHg}$ ) e conseqüentemente reduzindo as complicações inerentes ao teste (arritmias, acidose grave, hipocalemia, hipotensão arterial) e a perda do potencial doador durante o mesmo ${ }^{3}$. Se o ponto apnéico não for atingido neste intervalo de tempo, prolonga-se o período de observação para 10 minutos, conforme determina a legislação brasileira ${ }^{7,13}$.

\section{DISTÚRBIOS METABÓLICOS}

A Lei não faz nenhuma menção ao estudo do estado metabólico do paciente, apenas diz no Artigo $3^{\circ}$. da Resolução CFM no 1.480 de 08/08/97: "A morte encefálica deverá ser conseqüência de processo 
irreversível e de causa conhecida."

Entretanto pode ser observado Diabetes Insipidus em até $95 \%$ dos casos de morte encefálica, ocasionando hipernatremia na maioria dos pacientes ${ }^{14}$. A mortalidade entre os adultos com mais de 48 horas de hipernatremia aguda (sódio plasmático acima de $160 \mathrm{mEq} / \mathrm{litro}$ ) é de cerca de $60 \%$ dos pacientes. Foram observadas lesões neurológicas letais em pacientes com hiponatremia aguda sintomática (sódio plasmático inferior a $120 \mathrm{mEq} / \mathrm{litro})^{15}$.

A literatura internacional ${ }^{16}$ considera como critério de exclusão para a realização das provas de Morte Encefálica a presença de distúrbios eletrolíticos. Do ponto de vista prático, entretanto, um grande número de potenciais doadores possui distúrbio dos níveis de sódio e não estão internados em locais apropriados para a correção destas alterações.

\section{Proposta}

Os distúrbios hidroeletrolíticos devem ser um critério de exclusão para a realização de provas de morte encefálica quando se houver dúvidas que o distúrbio hidroeletrolítico possa estar piorando o estado de coma. Desta maneira, os pacientes com distúrbios hidroeletrolíticos graves que poderiam apresentar coma reversível então sendo preservados.

Consideramos que os pacientes com distúrbios hidroeletrolíticos podem realizar as provas de morte encefálica, incluindo o teste de apnéia, quando houver causa óbvia de coma irreversível ou lesão incompatível com a vida, como ferimento por projétil de arma de fogo transfixante do tronco, acidente vascular cerebral hemorrágico maciço ou exame confirmatório compatível com morte encefálica, pois desta maneira fica descaracterizada a correlação do distúrbio hidroeletrolítico com a etiologia do quadro clínico.

\section{HIPOTERMIA}

A existência da hipotermia é uma ocorrência comum em pacientes com morte encefálica. Entretanto, na grande maioria dos casos no Brasil ${ }^{18}$, trata-se de um fenômeno a posteriori.

Conforme já preconiza a lei, a presença de hipotermia deve ser excluída para que se faça o diagnóstico de morte encefálica.

\section{Proposta}

Inclusão dos pacientes hipotérmicos com causa óbvia de coma irreversível ou lesão incompatível com a vida nos protocolos de morte encefálica. Entretanto, durante os exames clínicos e complementares, a temperatura do paciente deve ser mantida acima de $36^{\circ} \mathrm{C}$.

\section{DOENÇA PRÉ-EXISTENTE}

A condição de candidato a doador pressupõe negatividade para doenças transmissíveis (infecciosas, detectadas por testes sorológicos padronizados) ou de potencial transmissão com o(s) órgão(s) transplantados. Neste sentido, Buell et al. ${ }^{17}$ referem a possibilidade de transplante de órgãos de pacientes portadores de neoplasias do sistema nervoso central, relatando uma taxa de 7\% de metastatização para o receptor, elevada a $53 \%$ em caso de quimioterapia prévia, presença de derivações ventrículo-peritoneal ou ventrículo-atrial, craniotomia prévia e alto grau de malignidade de gliomas. Entretanto, se houver aceitação, pelo receptor ou responsáveis, dos riscos acima mencionados, procede-se à captação dos órgãos e ao transplante, com o intuito de redução de demanda na lista de espera e melhora do quadro funcional do receptor, que deverá ser acompanhado evolutivamente.

\section{Proposta}

Procede-se ao protocolo de diagnóstico de morte encefálica e, em caso de aceitação pelo receptor, implanta-se o órgão.

Andrade AF de, Amorim RLO de, Paiva WS, Figueiredo EG, Barros e Silva LB de, Teixeira MJ. Clinical features of brain death - review and purposals. Rev Med (São Paulo). 2007 jul.set.;86(3):132-7.

ABSTRACT: Almost 70 thousand people await donation of organs in Brazil. The Hospital das Clínicas of the University of São Paulo is reference center in transplants of organs and woven and, at the same time that you/they take place surgeries of high complexity, it qualifies the professionals of health, being a facilitator for the integration of the same ones. Brain death today should be considered a medical emergency, because its diagnosis can save several lives. Its diagnosis is accomplished in agreement with the Brazilian Medicine Federal Council number $1.480 / 97$ and, based on that resolution and in our experience with whole transplant process, we presented in this paper proposed to avoid the potentials donors' loss.

KEY WORDS: Brain death/diagnosis. Organ transplantation/legislation and jurisprudence. Standards. 


\section{REFERÊNCIAS}

1. Wijdicks EFM. Clinical diagnosis and confirmatory testing of brain death in adults. In: Wijdicks EFM. Brain death. Philadelphia: Lippincott Willians \& Wilkins; 2001. p.61-90.

2. Jeret JS, Benjamin JL. Risk of hypotension during apnea testing. Arch Neurol. 1994;5:595-9.

3. Schaffer JA, Caronna JJ. Duration of apnea needed to confirm brain death. Neurology. 1978;28:661-6.

4. Coimbra CG. Implications of ischemic penumbra for the diagnosis of brain death. Braz $\mathrm{J}$ Med Biol Res. 1999;32(12):1479-87.

5. Hayashi N, Takasu, T. Brain ressucitation \& brain death -up-to-date 1998. Tokyo: University Research Center, Nihon University; 1998.

6. Ropper AM. Unusual spontaneous movements in brain death patients. Neurology. 1978;34:1089-92.

7. Silva SP. Morte encefálica. J CREMESP. 2001;165:3.

8. Mello LR. Ética na morte cerebral: pontos obscuros. Rev Soc Bras Neurocirur. 2001:10.

9. Andrade AF, Marino Jr R, Miura FK, Brock RS, Buchipguel C, Shu EBS. SPECT teste might precede apnea test in the diagnosis of brain death. In: $12^{\circ}$ Congresso Mundial de Neurocirurgia, Sidney, Austrália; 2001. Poster.

10. Wijdicks EFM. Brain death worldwide: accepted fact but no global consensus in diagnostic criteria. Neurology.
2002:20-5.

11. Schwarz G, Litscher G, Pfurtscheller G, Schalk HV, Rumpl E, Fuchs G. Brain death: timing of apnea testing in primary brain stem lesion. Intensive Care Med. 1992;18:315-6.

12. Ropper AM, Kennedy SK, Russel L. Apnea testing in the diagnosis of brain death. J Neurosurg. 1981;55: 942-6.

13. Dantas FO, Torres MP, Araújo S, Vieira RJ, Falcão ALE, Sardinha LAC, Terzi RGG. Morte encefálica: considerações gerais e protocolo para o seu diagnóstico. Rev Bras Ter Intens. 1992;4:50-4.

14. Wijdicks EFM. Pathophysiology responses to brain death. In: Brain death. Wijdicks EFM. Philadelphia: Lippincott Willians \& Wilkins; 2001. p. 29-43.

15. Santos BFC, Andrei AM, Rodrigues M. Distúrbios na concentração plasmática de sódio. In: Knobel E. Condutas no paciente grave. São Paulo: Atheneu; 1998. v.1, p.447-57.

16. Machado C. Guideliness for the determination of death. In: Machado C., editor Brain death. Amsterdam: Elsevier; 1995. p.75-80.

17. Buell JF, Trofe J, Sethuraman G, Hanaway MJ, Beebe TM, Gross TG, Alloway R, First MR, Woodle ES. Donors with central nervous system malignancies: are they truly safe? Transplantation. 2003;76(2): 340-3.

18. Teive HAG, Silvado CE. Conceito atual de morte. Rev Bras Neurol. 1991;27(4):133-6. 